\title{
Differential response to pulmonary rehabilitation in COPD: multidimensional profiling
}

\author{
Martijn A. Spruit ${ }^{1}$, Ingrid M.L. Augustin", Lowie E. Vanfleteren', Daisy J.A. Janssen", \\ Swetlana Gaffron ${ }^{2}$, Herman-Jan Pennings ${ }^{3}$, Frank Smeenk ${ }^{4}$, Willem Pieters ${ }^{5}$, \\ Jan J.A.M. van den Bergh ${ }^{6}$, Arent-Jan Michels ${ }^{7}$, Miriam T.J. Groenen ${ }^{1}$, \\ Erica P.A. Rutten ${ }^{1}$, Emiel F.M. Wouters ${ }^{1,8}$ and Frits M.E. Franssen ${ }^{1}$ on behalf of the \\ CIRO+ Rehabilitation Network
}

Affiliations: 'Dept of Research \& Education, CIRO+, Center of Expertise for Chronic Organ Failure, Horn, The Netherlands. ${ }^{2}$ Viscovery Software $\mathrm{GmbH}$, Vienna, Austria. ${ }^{3}$ Dept of Respiratory Medicine, St. Laurentius Hospital, Roermond, The Netherlands. ${ }^{4}$ Dept of Respiratory Medicine, Catharina Hospital, Eindhoven, The Netherlands. ${ }^{5}$ Dept of Respiratory Medicine, Elkerliek Hospital, Helmond, The Netherlands. ${ }^{6}$ Dept of Respiratory Medicine, St. Jans Gasthuis, Weert, The Netherlands. ${ }^{7}$ Dept of Respiratory Medicine, St Anna Hospital, Geldrop, The Netherlands. ${ }^{8}$ Dept of Respiratory Medicine, Maastricht University Medical Center (MUMC+), The Netherlands.

Correspondence: Martijn A. Spruit, CIRO, Dept of Research \& Education, Hornerheide 1, 6085 NM Horn, The Netherlands. E-mail: martijnspruit@ciro-horn.nl

ABSTRACT The aim of the present study was to profile a multidimensional response to pulmonary rehabilitation in patients with chronic obstructive pulmonary disease (COPD).

Dyspnoea, exercise performance, health status, mood status and problematic activities of daily life were assessed before and after a 40-session pulmonary rehabilitation programme in 2068 patients with COPD (mean forced expiratory volume in $1 \mathrm{~s}$ of $49 \%$ predicted). Patients were ordered by their overall similarity concerning their multidimensional response profile, which comprises the overall response on MRC dyspnoea grade, 6MWD, cycle endurance time, Canadian Occupational Performance Measure performance and satisfaction scores, Hospital Anxiety and Depression Scale anxiety and depression, and St George's Respiratory Questionnaire total score, using a novel non-parametric regression technique.

Patients were clustered into four groups with distinct multidimensional response profiles: $\mathrm{n}=378$ (18.3\%; "very good responder"), $\mathrm{n}=742$ (35.9\%; "good responder"), $\mathrm{n}=731$ (35.4\%; "moderate responder"), and $\mathrm{n}=217$ (10.5\%; "poor responder"). Patients in the "very good responder" cluster had higher symptoms of dyspnoea, number of hospitalisations $<12$ months, worse exercise performance, worse performance and satisfaction scores for problematic activities of daily life, more symptoms of anxiety and depression, worse health status, and a higher proportion of patients following an inpatient PR programme compared to the other three clusters.

A multidimensional response outcome needs to be considered to study the efficacy of pulmonary rehabilitation services in patients with COPD, as responses to regular outcomes are differential within patients with COPD.

@ERSpublications

Efficacy of pulmonary rehabilitation in patients with COPD needs to be assessed using a multidimensional response http://ow.ly/RsfYK

For editorial comments see Eur Respir J 2015; 46: 1538-1540 [DOI: 10.1183/13993003.01125-2015].

This article has supplementary material available from erj.ersjournals.com

Received: March 032015 | Accepted after revision: June 282015 | First published online: Oct 092015

Support statement: CIRO+ (Center of Expertise for Chronic Organ Failure) and Stichting "De Weijerhorst" financially supported this study.

Conflict of interest: Disclosures can be found alongside the online version of this article at erj.ersjournals.com

Copyright @ERS 2015 


\section{Introduction}

Pulmonary rehabilitation (PR) is a comprehensive intervention designed to improve the physical and psychological condition of people with chronic respiratory disease [1]. Daily symptoms, exercise performance and health status generally improve following PR [2]. Therefore, PR is recognised as a fundamental part of the integrated care of people with chronic obstructive pulmonary disease (COPD) [3].

Since financial resources for PR are often limited or even non-existing [4], identification of (clusters of) patients that do (or do not) respond to PR will become necessary in the near future to improve its cost-effectiveness. This requires consensus about the key performance measures of PR services. To date, changes in exercise performance and health status are often used to qualify individuals with COPD as responders or non-responders to PR [5-10]. Nevertheless, changes in the abovementioned outcomes following $\mathrm{PR}$ are mostly differential. Indeed, patients may improve health status without an improvement in exercise capacity or vice versa; and patients may improve walk distance without an improvement in cycle endurance or vice versa [8, 11-13]. So, the choice for exercise performance and/ or health status as key performance measures seems too simple, and the use of non-linear statistics seems inevitable. Moreover, multiple other outcomes (i.e., symptoms of dyspnoea, cycle endurance time, performance of problematic activities of daily life, and symptoms of anxiety and depression [2, 12, 14, 15]) have been identified by healthcare professionals as essential to evaluate the efficacy of PR services [4]. So, a comprehensive evaluation of the efficacy of PR in individuals with COPD is complex, and requires a thorough initial and outcome assessment $[1,16]$. Therefore, we sought to profile a multidimensional response to PR in patients with COPD, including symptoms of dyspnoea, exercise performance, health status, mood status, and problematic activities of daily life, using a non-parametric regression technique.

\section{Methods}

Patients

We extracted data from the Integrated Knowledge System based on BioXM ${ }^{\mathrm{TM}}$ (Biomax Informatics AG, Munich, Germany) of 3349 patients with the diagnosis of COPD who were evaluated during the initial assessment of a comprehensive PR programme at CIRO, centre of expertise for chronic organ failure in Horn (The Netherlands) between January 2006 to December 2012 [16]. Of these records, 706 patients dropped out during the $\mathrm{PR}$, while 575 patients had 5 or more missing values for the response indicators (please see below for more details) at baseline and/or outcome assessment. Finally, 2068 patients (42.9\% women) met the following inclusion criteria: a primary diagnosis of COPD, a post-bronchodilator forced expiratory volume in $1 \mathrm{~s} /$ forced vital capacity ratio of $\leqslant 0.70$, and completion of PR. This analysis also included patients with exacerbations prior to and/or during the study. Ethical approval was not indicated because all of the tests were done as part of the routine initial assessment [16], and analysed retrospectively. The Board of Directors of CIRO approved the use of de-identified patients' records.

\section{Testing}

As part of routine 3-day initial assessment [16], patients underwent, amongst other tests and questionnaires, a maximal incremental cycle test during which peak work rate was determined. Subsequently, on a different day, patients performed a constant work rate test at $75 \%$ of the determined peak work rate [17]. Patients also performed two 6-min walk tests [18] and the test with the longest 6-min walk distance $(6 \mathrm{MWD})$ was used for further analysis $[19,20]$. Spirometry, physical examination, medical history and Medical Research Council (MRC) dyspnoea scale data were obtained. Patients underwent an intake by an occupational therapist, including the Canadian Occupational Performance Measure (COPM) to identify and discuss specific problematic activities of daily life [21]. In addition, patients identified their perception of how well they were performing the problematic activities of daily life (performance score; COPM-P) and how satisfied they were with this level of performance (satisfaction score; COPM-S). These scores were ascertained by using the cue cards to identify a score between 1 ("not able to do it" or "not at all satisfied", respectively) to 10 points ("able to do it extremely well" or "extremely satisfied"). The COPM is reliable in COPD [22] and responsive to PR [15]. Mood status has been assessed using the Hospital Anxiety and Depression Scale (HADS) [23]. HADS is divided in an anxiety subscale (HADS-A) and a depression subscale (HADS-D). Total scores for each subscale can range from 0 (optimal) to 21 (worst) points. A score from 8 to 10 indicates a mild mood disturbance, a score from 11 to 14 a moderate mood disturbance and a score from 12 to 21 a severe mood disturbance [23]. For assessment of disease-specific health status, the St. George Respiratory Questionnaire (SGRQ) has been used. The SGRQ consists of 50 items, divided in three domains (symptoms, activities and impact), providing three domain scores. A total score is also provided (SGRQ-T). Scores can range from 0 (optimal) to 100 points (worst) [24]. 


\section{Intervention}

All patients underwent $\mathrm{PR}$, as described previously [25]. In brief, CIRO+ provides a state-of-the-art interdisciplinary PR programme for patients with COPD consisting of 40 sessions, in line with the 2013 American Thoracic Society/European Respiratory Society Statement on PR [1]. PR can be inpatient ( 8 weeks, 5 days.week ${ }^{-1}$ ) or outpatient ( 8 weeks, 3 half days.week ${ }^{-1}$, followed by 8 weeks 2 half days.week $\left.{ }^{-1}\right)$. The outpatient PR programmes took place in the CIRO+ rehabilitation network. During baseline assessment, a careful characterisation of the extra-pulmonary features of patients with COPD was performed, which determined the application of various treatments: physical exercise training, occupational therapy, nutritional counselling, psychosocial counselling, education and exacerbation management. Physical exercise training was the cornerstone of the programme, consisting of strengthening exercises, treadmill walking and stationary cycling. All exercises were performed at moderate-to-high intensity to obtain an overload stimulus. Moreover, the training intensity increased during the rehabilitation period, based on dyspnoea and fatigue symptom scores. All patients underwent flexibility exercises, general physical exercise for lower and upper extremities, and daily supervised 30-min outdoor walks. Patients, who were too dyspnoeic to perform endurance/interval/resistance training, received lower-limb high-frequency neuromuscular electrical stimulation [26].

\section{Statistics}

Data are presented as mean \pm SD or as frequency, as appropriate. Moreover, the patient data were ordered based on the overall similarity concerning selected attributes. The attributes that drove the ordering process of the patients in the map are the overall response and the differences in the response indicators. The overall response is based on the weighted standardised differences between initial and outcome assessment of all eight response indicators: MRC, $6 \mathrm{MWD}$, cycle endurance time, COPM-P, COPM-S, HADS-A, HADS-D, and SGRQ-T. Thus, patients with a similar response profile are placed closed to each other in the map. Based on the ordering of the patients in the map, the hierarchical ward cluster algorithm has been applied, to cluster the patients into four response clusters. The values of all attributes included in the analyses could then be recalled cluster by cluster to be exported for the statistical tables. For the clustering of patients, the Viscovery Data Mining Suite, version 6.1 by Viscovery Software GmbH (Vienna, Austria; www.viscovery.net) was used, which is based on the technology of self-organising maps (SOMs, also referred to as Kohonen maps). SOMs represent an ordered representation of multi-dimensional data which simplifies complexity and reveals meaningful relationships, and have been used before in COPD [27].

Four clusters of patients with substantially different response profiles have been generated. The efficacy of the pulmonary rehabilitation programme has been evaluated based on the minimal clinically important difference (MCID). The following MCIDs were used: -1 grade on MRC dyspnoea scale [8]; $+30 \mathrm{~m}$ on 6 MWD $[19,20] ;+100 \mathrm{~s}$ on cycle endurance time [13]; +2 points on COPM-P [15]; +2 points on COPM-S [15]; -1.5 points on HADS-A [28]; -1.5 points on HADS-D [28]; and -4 points on SGRQ-T [29].

Please see online supplement for all details on the statistics.

\section{Results}

\section{Baseline characteristics}

On average, patients had moderate-to-very severe COPD, an impaired exercise capacity, a poor health status, and experienced problems during the performance of activities of daily life. Moreover, patients were on multiple pulmonary and non-pulmonary drug treatments (table E1 in the supplementary material). Patients with long-term oxygen therapy generally had more symptoms of dyspnoea, and worse exercise performance, mood status and health status compared with patients without long-term oxygen therapy (table 1).

\section{Response to pulmonary rehabilitation: whole group}

Significant improvements were found for symptoms of dyspnoea (MRC: $-0.4 \pm 1.1), 6 \mathrm{MWD}(27 \pm 57 \mathrm{~m})$, cycle endurance time $(208 \pm 328 \mathrm{~s})$, performance of problematic activities of daily life (COPM-P: $2.0 \pm 1.7$ points), the satisfaction with the performance of the problematic activities of daily life (COPM-S: $2.6 \pm 2.1$ points), symptoms of anxiety (HADS-A: $-1.4 \pm 3.5$ points), symptoms of depression (HADS-D: $-1.4 \pm 3.5$ points), and health status (SGRQ total score: $-5.3 \pm 12.6$ points) (all $\mathrm{p}<0.01$ ).

\section{Multidimensional response profiling: whole sample}

The 2068 patients with COPD were clustered into four groups with distinct multidimensional response profiles: $n=378$ (18.3\%) in the cluster "very good responder", $n=742$ (35.9\%) in the cluster "good responder", $\mathrm{n}=731$ (35.4\%) in the cluster "moderate responder", and $\mathrm{n}=217(10.5 \%)$ in the cluster "poor responder" (table 2). The response to $\mathrm{PR}$ was best in the very good responder cluster on all outcome measures compared with the other clusters (table 2, figure 1). Indeed, a clinically relevant improvement 
TABLE 1 Baseline characteristics

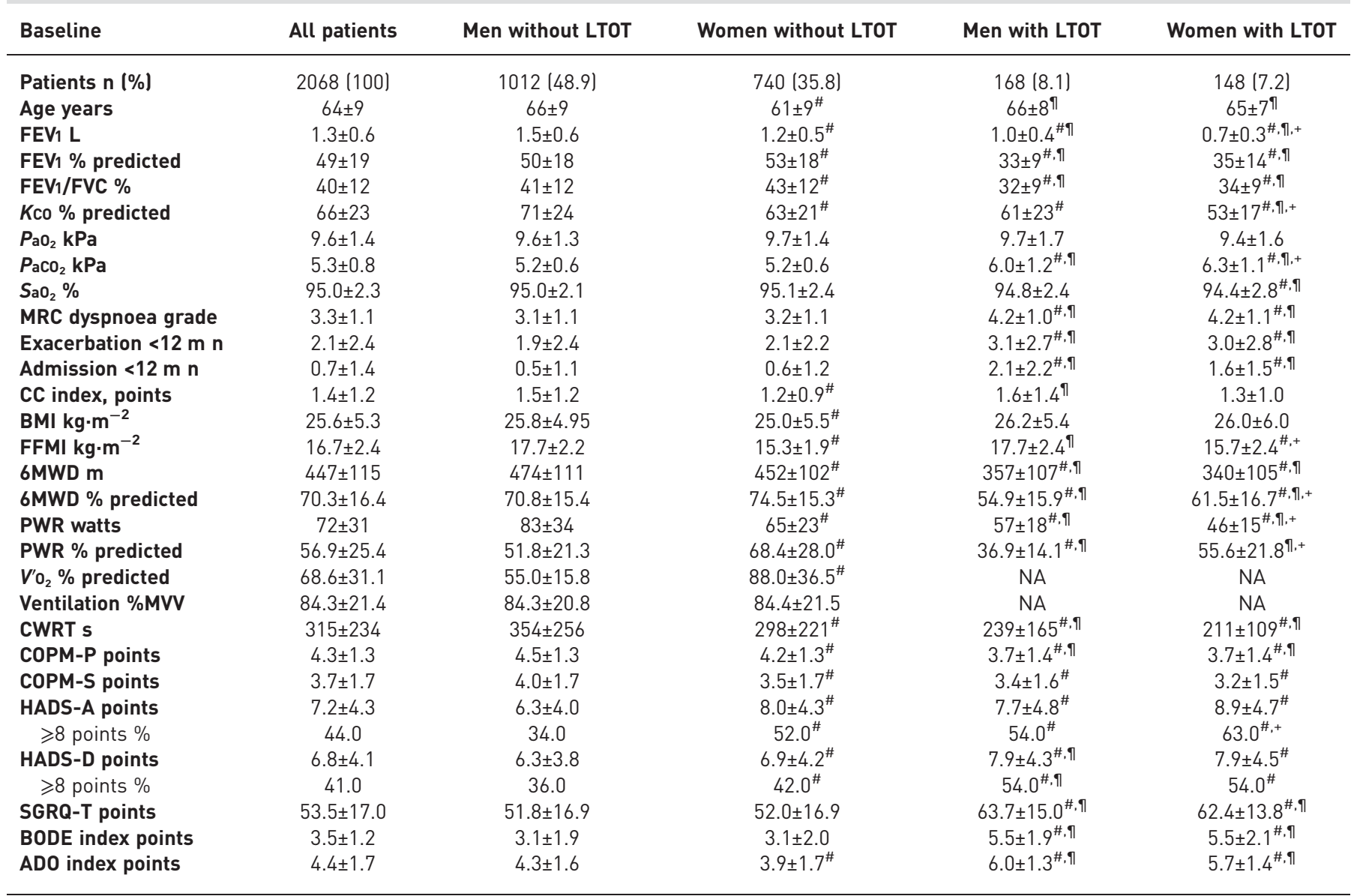

Data are presented as mean \pm SD, unless otherwise stated. LTOT: long-term oxygen therapy; FEV1: forced expiratory volume in $1 \mathrm{~s}$; FVC: forced vital capacity; $K \mathrm{Co}$ : transfer factor of the lung for carbon monoxide; $\mathrm{PaO}_{2}$ : arterial oxygen tension; $\mathrm{PaCO}_{2}$ : $\mathrm{SaO}_{2}$ : arterial oxygen saturation; arterial carbon dioxide tension; MRC: Medical Research Council; CC index: Charlson Comorbidity index; BMI: body mass index; FFMI: fat-free mass index; 6MWD: 6-min walk distance; PWR: peak work rate; $V^{\prime} \mathrm{O}_{2}$ : oxygen uptake; MVV: maximal voluntary ventilation; CWRT: constant work-rate test; COPM-P: Canadian Occupational Performance Measure, performance score; COPM-S: Canadian Occupational Performance Measure, satisfaction score; HADS-A: Hospital Anxiety and Depression Scale, anxiety scores; HADS-D Hospital Anxiety and Depression Scale, depression scores; SGRQ-T: St. George's Respiratory Questionnaire, total score; BODE: body mass index, airflow obstruction, dyspnoea, exercise capacity; ADO: age, dyspnoea, airflow obstruction; NA: Not assessed. Missing data in men without long-term oxygen therapy were MRC, $n=33 ; 6 \mathrm{MWD}$, $\mathrm{n}=43 ; V^{\prime} \mathrm{O}_{2}, \mathrm{n}=47$; ventilation, $\mathrm{n}=47$; CWRT, $\mathrm{n}=110$; COPM-P, $\mathrm{n}=106$; COPM-S, $\mathrm{n=106}$; HADS-A, $\mathrm{n}=34$; HADS-D, $\mathrm{n}=34 ;$ SGRQ-T, $\mathrm{n}=71$. Missing data in women without long-term oxygen therapy: MRC, $n=15 ; 6 M W D, n=33 ;$ VO2, $n=49$; ventilation, $n=49 ;$ CWRT, $n=75 ; C O P M-P, n=58 ; C O P M-S, n=58$; HADS-A, $n=31$; HADS-D, $n=31$; SGRQ-T, $n=53$. Missing data in men with long-term oxygen therapy: MRC, $n=2 ; 6 M W D, n=7 ; C W R T, n=19 ; C O P M-P$, $\mathrm{n}=12$; COPM-S, $n=12$; HADS-A, $n=11$; HADS-D, $n=11$; SGRQ-T, $n=21$. Missing data in women with long-term oxygen therapy: MRC, $n=1 ; 6 M W D$, $n=7$; CWRT, $n=34$; COPM-P, $n=8$; COPM-S, $n=8$; HADS-A, $n=12$; HADS-D, $n=12$; SGRQ-T, $n=24$. * : $p<0.01$ versus men without long-term oxygen therapy; ${ }^{\uparrow}: p<0.01$ versus women without long-term oxygen therapy; ${ }^{+}: p<0.01$ versus men with long-term oxygen therapy.

was achieved in $85 \%$ of the outcomes of the patients in cluster "very good responder", while this was only achieved in $11 \%$ of the outcome of the patients in poor responder cluster (table 2, figure 2).

\section{Cluster characteristics: whole sample}

Table 3 summarises the baseline characteristics of the patients after stratification for the multidimensional response clusters. Age, the proportion of women, the degree of airflow limitation and the transfer factor for carbon monoxide were comparable between clusters. Patients in the very good responder cluster had significantly higher symptoms of dyspnoea, number of hospital admissions in the last 12 months, a worse exercise performance, worse performance and satisfaction scores for problematic activities of daily life, more symptoms of anxiety and depression, a worse health status, and a higher proportion of patients following an inpatient PR programme compared with the other three clusters. Moreover, patients from the very good responder cluster had a higher proportion of long-term oxygen therapy users, a higher body mass index and a higher fat-free mass index at baseline compared with the patients of the moderate responder and poor responder clusters. 
TABLE 2 Outcomes of pulmonary rehabilitation

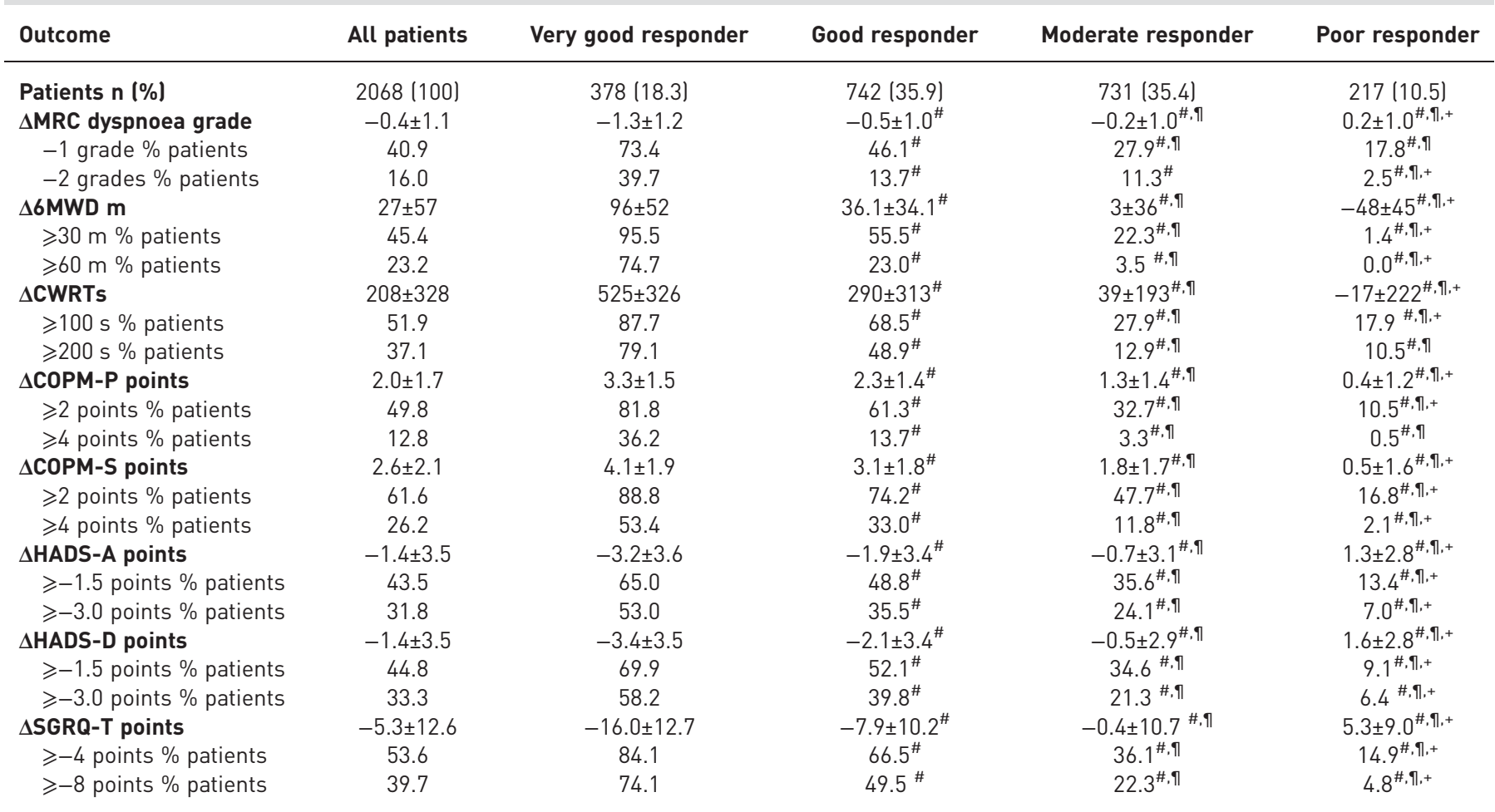

Data are presented as mean \pm SD, unless otherwise stated. $\Delta$ : change; MRC: Medical Research Council; 6MWD: 6-min walk distance; CWRT: constant work-rate test; COPM-P: Canadian Occupational Performance Measure, performance score; COPM-S: Canadian Occupational Performance Measure, satisfaction score; HADS-A: Hospital Anxiety and Depression Scale, anxiety scores; HADS-D- Hospital Anxiety and Depression Scale, depression scores; SGRQ-T: St. George's Respiratory Questionnaire, total score. Missing data: MRC, $n=988 ; 6 M W D, n=107$; CWRT, $n=279$; COPM-P, $n=228$; COPM-S, $n=228$; HADS-A, $n=240$; HADS-D, $n=240$; SGRQ-T, $n=449$. \# : $p<0.01$ versus cluster very good responder cluster; ${ }^{\text {ๆ: }} p<0.01$ versus good responder cluster; ${ }^{+}: p<0.01$ versus moderate responder cluster.

Multidimensional response profiling, after stratification for sex and use of long-term oxygen therapy The current sample consisted of 1012 male and 740 female COPD patients without long-term oxygen therapy, and 168 male and 148 female COPD patients with long-term oxygen therapy. These four subgroups were also divided up into four clusters with distinct multidimensional response profiles (see tables E2 to E5 and figures E1 to E4 in the online supplementary material for all details). The response to PR was best in the very good responder cluster on all outcome measures compared with the other clusters, irrespective of sex and the use of long-term oxygen therapy.

\section{Cluster characteristics after stratification for sex and use of long-term oxygen therapy}

Tables E6 to E9 in the online supplementary material summarise the baseline characteristics of the four clusters of each subgroup. In brief, in the male patients without long-term oxygen therapy (table E6), baseline scores for problematic activities of daily life, symptoms of anxiety and depression, and health status were significantly worse in the very good responder cluster compared to the other clusters. The degree of dyspnoea and the 6MWD was significantly worse in the patients of the very good responder cluster compared with the good responder or moderate responder clusters, but were comparable to values of the patients of the poor responder cluster.

In the female patients without long-term oxygen therapy (table E7), the patients in the very good response cluster had a significantly higher baseline degree of dyspnoea, and worse health status compared with the other three clusters. Moreover, baseline 6MWD was lower compared with moderate response and poor response clusters.

In the male patients with long-term oxygen therapy (table E8), the patients in the poor response cluster had a significantly lower body mass index and fat-free mass index compared with the other three clusters. Moreover, the patient in the very good response cluster had a significantly worse health status compared to the patients of the moderate response and poor response clusters. 


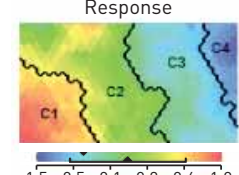

b) Outcomes $\geqslant 1 \times M C I D \%$

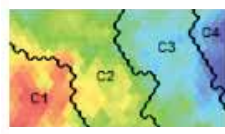

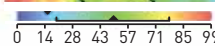

c)

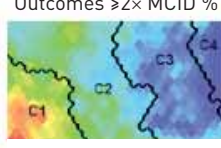

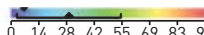

$\triangle M R C$ grade

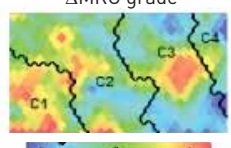

-1.0-0.30.20.71.32.

$\triangle \mathrm{MRC} \geqslant 1$ grade

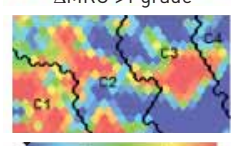

\begin{tabular}{llllll}
\hline \hline 0 & 0.2 & 0.4 & 0.6 & 0.8 & 1.0
\end{tabular}

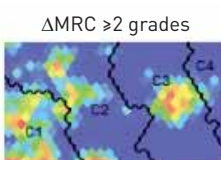

D. $\triangle 6 M W D \mathrm{~m}$

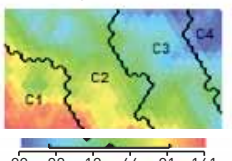

$\triangle 6 \mathrm{MWD} \geqslant 30 \mathrm{~m}$

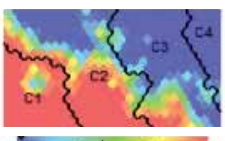

$\begin{array}{llllll}0 & 0.2 & 0.4 & 0.6 & 0.8 & 1.0\end{array}$

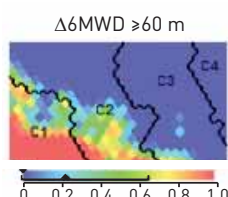

$\triangle C W R T$ s

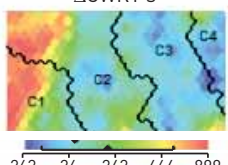

$\triangle C W R T \geqslant 100 \mathrm{~s}$

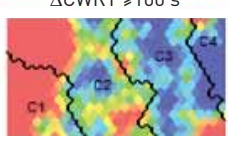

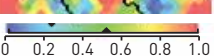

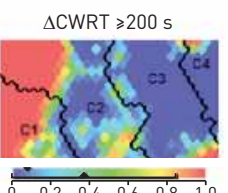

$\triangle C O P M-P$ points

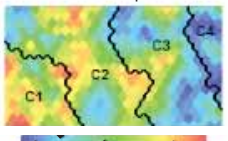

$\frac{5.9}{0.91 .72 .53 .450}$

$\triangle C O P M-P \geqslant 2$ points

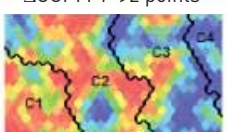

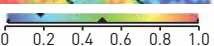

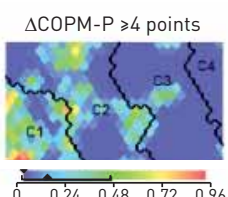

$\triangle$ COPM-S points

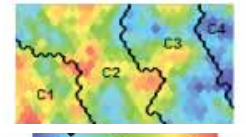

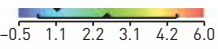

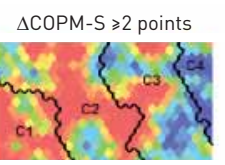

$=\frac{h}{0.20 .60 .810}$

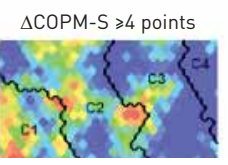

. $\triangle$ HADS-A points

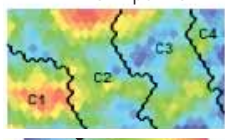

年,

$\triangle H A D S-A \geqslant 1.5$ points

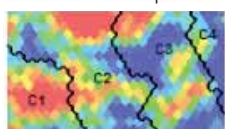

$\frac{2.4}{0.140 .6018}$

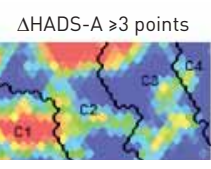

C. $<2$

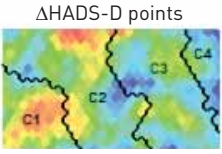

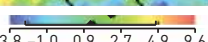
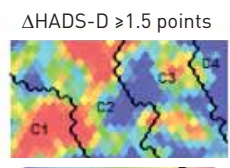

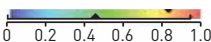

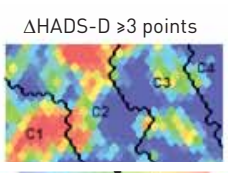

Berang

$\triangle S G R Q-T$ points

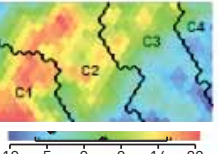

$\triangle S G R Q-T \geqslant 4$ points

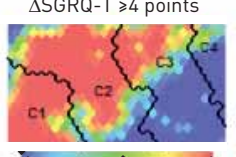

\begin{tabular}{lllllll} 
& \\
\hline & 0.2 & 0.4 & 0.6 & 0.8 & 1.0
\end{tabular}

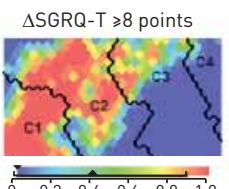

FIGURE 1 Panels generated using Viscovery (Viscovery Software GmbH, Vienna, Austria). The Viscovery program placed all patients on a specific position on all maps based on their multidimensional response profile. The more subjects resemble in terms of their response to pulmonary rehabilitation the closer they are on the map. Contrarily, the more they differ the further they are away from each other. When looking at an outcome measure of pulmonary rehabilitation, patients "raise a red flag" if they had a very good response, "a green flag" when the response was good to moderate, and "a blue flag" when the response was poor. In this way the maps can be interpreted. Using the topology of the self-organising map, the Viscovery program could identify four different clusters of patients with chronic obstructive pulmonary disease (COPD) with a significantly different multidimensional response profile. C1: cluster "very good responder" ( $n=378$ ); C2: cluster 2 "good responder" (n=742); C3: cluster 3 "moderate responder" (n=731); C4: cluster 4 "poor responder" (n=217). a) Left panel shows the multidimensional response outcome. All other attribute pictures in a) are the absolute change in Medical Research Council (MRC) dyspnoea grade, 6-min walk distance (6MWD), cycle endurance time (constant work-rate test; CWRT). Canadian Occupational Performance Measure, Performance (COPM-P), Canadian Occupational Performance Measure, Satisfaction (COPM-S), Hospital Anxiety and Depression Scale, anxiety (HADS-A), Hospital Anxiety and Depression Scale, depression (HADS-D), and St. George's Respiratory Questionnaire total score (SGRQ-T). b) Left panel shows the proportion of clinically relevant outcomes (exceeding at least $1 \times$ minimally clinical important difference (MCID)). All other panels are the proportion of patients per outcome showing a clinically relevant improvement (exceeding at least $1 \times$ MCID). c) First panel shows the proportion of clinically relevant outcomes (exceeding at least $2 \times$ $\mathrm{MCID}$. All other panels are the proportion of patients per outcome showing a clinically relevant improvement (exceeding at least $2 \times \mathrm{MCID}$ ). 

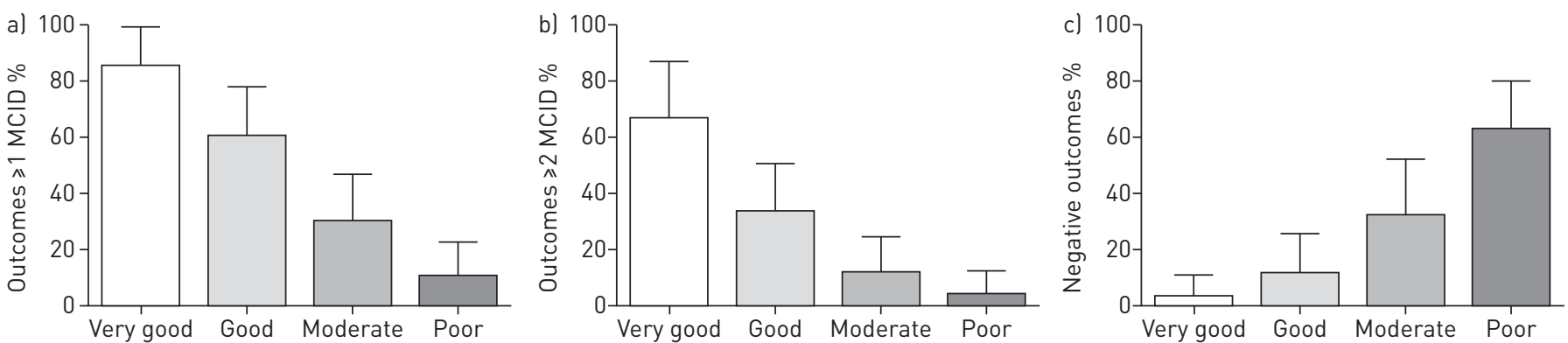

FIGURE 2 Proportion of outcomes which exceed the pre-defined minimal clinically important difference (MCID) at least a) once or b) twice, or c) have negative outcomes, in the very good, moderate and poor responders.

In the female COPD patients with long-term oxygen therapy (table E9), baseline 6-min walk distance was significantly lower in the patients of the very good response cluster compared with the other three clusters. Moreover, the patients in the poor response cluster had a significantly better satisfaction scores for the problematic activities of daily life compared to the other three clusters.

\section{Discussion}

The current findings corroborate that responses to regular PR outcomes are differential in a large sample of patients with COPD. Moreover, this is the first study to show that patients with COPD can be clustered based on their multidimensional response to a comprehensive PR programme, identifying groups of patients with a very good, good, moderate or poor response.

\section{Differential response to $P R$}

Generally, PR is beneficial for adults with chronic respiratory disease, including COPD [1]. Nevertheless, the response to PR may vary considerably between patients with COPD $[5,8,9,14,30,31]$. Moreover, individual patients respond differential on various types of outcome measures $[8,11]$. The current results in a convenience sample of 2068 well-characterised patients with COPD corroborate these findings (figure 1). These findings emphasise that key performance measures to evaluate the efficacy of PR in patients with COPD have to be chosen very carefully and should focus on multiple domains. The choice for exercise performance and/or health status as key performance measure is too simple. Indeed, to better understand the response to PR in patients with COPD, non-linear statistics were needed. The unbiased approach to cluster patients based on their multidimensional response in a large sample of well-characterised patients with COPD is a major strength of the current analyses. Indeed, the use of Viscovery SOMs allows detailed insight in the differential responses to PR (figure 1). This is a true novelty of the current analyses.

\section{Clinically relevant improvements}

In the very good responder cluster, the minimal clinically relevant improvement was achieved in $85 \%$ of the outcomes at least once and, in $67 \%$ of the outcomes, at least twice (table 2; figure 1). These findings suggest that the patients with COPD in the very good responder cluster are truly benefiting from PR, on (almost) all domains. Moreover, patients in the other clusters also still achieved a clinically relevant improvement in $60 \%$ (good responder cluster), 30\% (moderate responder cluster) and 11\% of the outcomes (poor responder cluster) (table 2). These findings prove again the clinical value of PR in patients with COPD who are still symptomatic even though they did receive the optimal medical care before enrolment. The fact that patients in the poor response cluster still have some clinically relevant improvements in individual outcomes also confirms, that we have to be very careful in defining key outcome measures of PR.

\section{Poor response to pulmonary rehabilitation}

The poor response in a subgroup of patients with COPD (figure 2) may be surprising at first sight, as PR provides a comprehensive approach. Then again, the heterogeneity in pulmonary and extra-pulmonary features [32-34] is a clinical challenge to personalise PR programmes for patients with COPD. Consequently, not all patients with COPD are expected to benefit from PR $[5,8,9,14,30,31]$. These are clinically relevant observations, as patients, members from their social circle, healthcare professionals, policy makers and payers have a clear interest in the cost-effectiveness of interventions related to the integrated care of patients with COPD. So, to provide true transparency to its main stakeholders, PR services need to give detailed insights in the efficacy of pulmonary rehabilitation on the individual outcome measures, as well as in a multidimensional outcome measure. 
TABLE 3 Baseline characteristics after stratification for multidimensional response clusters

\begin{tabular}{|c|c|c|c|c|}
\hline Baseline & $\begin{array}{l}\text { Very good } \\
\text { responder }\end{array}$ & $\begin{array}{l}\text { Good } \\
\text { responder }\end{array}$ & $\begin{array}{l}\text { Moderate } \\
\text { responder }\end{array}$ & $\begin{array}{c}\text { Poor } \\
\text { responder }\end{array}$ \\
\hline Patients n (\%) & $378(18.3)$ & $742(35.9)$ & $731(35.4)$ & $217(10.5)$ \\
\hline Age years & $62.9 \pm 8.8$ & $63.7 \pm 9.0$ & $64.2 \pm 8.7$ & $64.4 \pm 9.1$ \\
\hline Sex \% women & 41.8 & 43.9 & 42.7 & 42.4 \\
\hline FEV1 L & $1.31 \pm 0.64$ & $1.31 \pm 0.54$ & $1.31 \pm 0.57$ & $1.27 \pm 0.56$ \\
\hline FEV $1 \%$ predicted & $47.4 \pm 20.2$ & $48.9 \pm 17.8$ & $48.8 \pm 18.3$ & $47.9 \pm 18.8$ \\
\hline Kco \% predicted & $67.7 \pm 22.7$ & $67.0 \pm 23.8$ & $64.9 \pm 21.9$ & $64.1 \pm 22.2$ \\
\hline LTOT use $\%$ patients & 21.7 & 15.9 & $12.2^{\#}$ & $12.4^{\#}$ \\
\hline $\mathrm{PaO}_{2} \mathrm{kPa}$ & $9.6 \pm 1.4$ & $9.7 \pm 1.4$ & $9.6 \pm 1.3$ & $9.7 \pm 1.3$ \\
\hline $\mathrm{PaCO}_{2} \mathrm{kPa}$ & $5.2 \pm 0.7$ & $5.2 \pm 0.6$ & $5.2 \pm 0.6$ & $5.3 \pm 0.8$ \\
\hline $\mathrm{SaO}_{2} \%$ & $94.9 \pm 2.6$ & $95.0 \pm 2.4$ & $95.1 \pm 2.1$ & $95.0 \pm 2.1$ \\
\hline MRC grade & $3.7 \pm 1.1$ & $3.3 \pm 1.1^{\#}$ & $3.2 \pm 1.1^{\#}$ & $3.2 \pm 1.1^{\#}$ \\
\hline Exacerbation $<12 \mathrm{~m} \mathrm{n}$ & $2.5 \pm 2.6$ & $2.1 \pm 2.5$ & $2.0 \pm 2.4^{\#}$ & $2.0 \pm 1.9$ \\
\hline Admission < $12 \mathrm{~m} \mathrm{n}$ & $1.1 \pm 1.8$ & $0.7 \pm 1.2^{\#}$ & $0.6 \pm 1.3^{\#}$ & $0.7 \pm 1.3^{\#}$ \\
\hline $\mathrm{CC}$ index points & $1.4 \pm 1.2$ & $1.4 \pm 1.2$ & $1.4 \pm 1.1$ & $1.4 \pm 1.1$ \\
\hline BMI $\mathrm{kg} \cdot \mathrm{m}^{-2}$ & $26.3 \pm 5.6$ & $25.9 \pm 5.5$ & $25.1 \pm 5.0^{\#, 9}$ & $24.8 \pm 4.6^{\#, 9}$ \\
\hline $\mathrm{FFMI} \mathrm{kg} \cdot \mathrm{m}^{-2}$ & $17.1 \pm 2.7$ & $16.8 \pm 2.4$ & $16.6 \pm 2.3^{\#}$ & $16.5 \pm 2.2^{\#}$ \\
\hline 6MWD m & $405 \pm 123$ & $452 \pm 113^{\#}$ & $461 \pm 112^{\#}$ & $457 \pm 104^{\#}$ \\
\hline 6MWD \% predicted & $63.3 \pm 17.4$ & $71.4 \pm 15.6^{\#}$ & $72.3 \pm 16.0^{\#}$ & $71.7 \pm 15.7^{\#}$ \\
\hline PWR watts & $68.2 \pm 32.3$ & $73.5 \pm 31.4$ & $72.9 \pm 30.5$ & $70.4 \pm 28.3$ \\
\hline PWR \% predicted & $50.5 \pm 22.7$ & $59.1 \pm 27.0^{\#}$ & $57.7 \pm 24.3^{\#}$ & $57.3 \pm 26.3^{\#}$ \\
\hline $\mathrm{V}^{\prime} \mathrm{O}_{2} \%$ predicted & $64.2 \pm 24.6$ & $70.5 \pm 32.7$ & $68.3 \pm 31.1$ & $69.8 \pm 34.1$ \\
\hline Ventilation \%MVV & $84.3 \pm 22.3$ & $84.0 \pm 21.2$ & $83.9 \pm 20.8$ & $87.2 \pm 22.6$ \\
\hline CWRT s & $295 \pm 173$ & $320 \pm 225$ & $326 \pm 265$ & $296 \pm 238$ \\
\hline COPM-P points & $3.8 \pm 1.3$ & $4.2 \pm 1.3^{\#}$ & $4.5 \pm 1.3^{\#, 9}$ & $4.5 \pm 1.4^{\#, \eta}$ \\
\hline COPM-S points & $3.2 \pm 1.6$ & $3.6 \pm 1.7^{\#}$ & $4.0 \pm 1.7^{\#, 9}$ & $4.1 \pm 1.8^{\# . \rrbracket}$ \\
\hline HADS-A points & $8.4 \pm 4.3$ & $7.2 \pm 4.2^{\#}$ & $6.8 \pm 4.3^{\#}$ & $6.3 \pm 4.3^{\# .9}$ \\
\hline$\geqslant 8$ points $\%$ patients & $\begin{array}{l}5.41 .0 \\
57.0\end{array}$ & $45.0^{\#}$ & $38.0^{\#, 9}$ & $36.0^{\#}$ \\
\hline HADS-D points & $8.0 \pm 4.1$ & $6.7 \pm 4.0^{\#}$ & $6.4 \pm 4.0^{\#}$ & $5.9 \pm 3.9^{\#, \pi}$ \\
\hline$\geqslant 8$ points $\%$ patients & 55.0 & $40.0^{\#}$ & $36.0^{\#}$ & $32.0^{\#}$ \\
\hline SGRQ points & $61.5 \pm 15.2$ & $53.6 \pm 16.5^{\#}$ & $50.2 \pm 17.1^{\#, \pi}$ & $50.4 \pm 17.0^{\#}$ \\
\hline BODE index points & $4.0 \pm 2.3$ & $3.4 \pm 2.1^{\#}$ & $3.3 \pm 2.1^{\#}$ & $3.4 \pm 2.0^{\#}$ \\
\hline ADO index points & $4.7 \pm 1.8$ & $4.3 \pm 1.8^{\#}$ & $4.3 \pm 1.6^{\#}$ & $4.4 \pm 1.7$ \\
\hline Inpatient/outpatient \% & $64 / 36$ & $41 / 59^{\#}$ & $31 / 69^{\#, \pi}$ & $25 / 75^{\#, 9}$ \\
\hline
\end{tabular}

Data are presented as mean $\pm S D$, unless otherwise stated. $F E V 1$ : forced expiratory volume in $1 \mathrm{~s} ; \mathrm{Kco}$ : transfer factor of the lung for carbon monoxide; $\mathrm{PaO}_{2}$ : arterial oxygen tension; $\mathrm{PaCO}_{2}$ : arterial carbon dioxide tension; $\mathrm{SaO}_{2}$ : arterial oxygen saturation; MRC: Medical Research Council; CC index: Charlson Comorbidity index; BMI: body mass index; FFMI: fat-free mass index; 6MWD: 6-min walk distance; PWR: peak work rate; $V^{\prime} \mathrm{O}_{2}$ : oxygen uptake; MVV: maximal voluntary ventilation; CWRT: constant work-rate test; COPM-P: Canadian Occupational Performance Measure, performance score; COPM-S: Canadian Occupational Performance Measure, satisfaction score; HADS-A: Hospital Anxiety and Depression Scale, anxiety scores; HADS-D Hospital Anxiety and Depression Scale, depression scores; SGRQ: St George's Respiratory Questionnaire; BODE: body mass index, airflow obstruction, dyspnoea, exercise capacity; ADO: age, dyspnoea, airflow obstruction. \# : $p<0.01$ versus very good responder cluster; ๆ: $p<0.01$ versus good responder cluster. No statistically significant differences in baseline characteristics between moderate responder and poor responder clusters.

The poor response to rehabilitation in a subgroup of patients does not seem to be COPD-specific and/or rehabilitation-specific. Indeed, also subgroups of patients with chronic neurological diseases [35], chronic cardiac diseases [36], or chronic musculoskeletal diseases [37] respond poorly to specialised rehabilitative interventions. Moreover, response to pharmacological therapy [38, 39], ambulatory oxygen therapy [40], bronchoscopic interventions [41] and lung volume reduction surgery [42] is also poor in subgroups of patients with COPD. These findings emphasise the need for a personalised approach of patients with chronic conditions, and the awareness that a "one size fits all" approach will not result in optimal chronic disease management [43].

\section{Response prediction}

It was beyond the aim of the current study to predict response based on the baseline characteristics. Nevertheless, it seems difficult to predict at the start of the programme who will end up in which cluster, as only $22.2 \%, 6.5 \%$ or $2.8 \%$ of the baseline values differed significantly $(\mathrm{p}<0.01)$ between the poor 
responder cluster and the very good responder, good responder and moderate responder clusters, respectively (tables E6 to E9). The analyses do emphasise that sex, age and the degree of airflow limitation cannot be used to identify possible responders (or non-responders) to PR as these were comparable between response clusters. Moreover, the baseline mean Charlson comorbidity score was comparable between the response clusters (table 3). These findings suggest that self-reported comorbidities generally do not influence the multidimensional response to PR. Recently, MesquiTA et al. [44] also showed that changes in exercise performance and health status were not affected by comorbidities that were based on objective measurements.

\section{Methodological considerations}

The PR programme at CIRO+ is executed according to the 2013 ATS/ERS Statement on Pulmonary Rehabilitation [1], and provided by a skilled and dedicated team. Nevertheless, individual programmes most probably varied between patients based on the results of the initial assessment [16]. Indeed, the key to success may, at least in part, be hidden in the actual content of the PR programme. Detailed information on the exact content of the individual programme is lacking in the current study. Therefore, the present results are hypothesis-generating rather than definitive. Interestingly, the proportion of outpatients increased while the multidimensional response to PR worsened, in particular in the patients without long-term oxygen therapy (tables E6 and E7). Whether and to what extent these differences are due to the PR setting (inpatient versus outpatient) and/or the frequency of the programme (5 days per week for 8 weeks versus 3 days per week for 8 weeks followed by 2 days per week for 8 weeks) remains to be determined in a randomised controlled trial.

The current multidimensional response profiling was based on eight outcome measures, including two types of functional exercise performance, health status, mood status, a situational measure of dyspnoea, and problematic activities of daily life, which were identified by healthcare professionals as essential [4]. Obviously, other PR outcome measures, such as physical activity, self-efficacy and disease-specific knowledge, may also be of interest for patients with COPD [1]. The current statistical approach allows the addition of other outcome measures to the multidimensional response profiling. Future, prospective studies need to apply their own multidimensional response profiling and, in turn, should try to corroborate the current cluster findings. Obviously, to enable concise statistical analyses at any point in time, the entire PR process (e.g., referral, baseline assessment, the rehabilitative interventions, short-term outcome assessment and follow up) must be managed and monitored by appropriate information and computer technology infrastructure. Indeed, it may even be a critical success factor for chronic disease management in general, and $\mathrm{PR}$ in particular.

To conclude, the current study is the first to profile the multidimensional response to PR using a non-parametric regression technique. The current approach allows us to cluster patients with COPD into groups, and, in turn, identify who benefits most or least from PR after completion of the programme. For the poor responders, we may need to redesign ongoing PR programmes. The current results are the next step in providing detailed insights in the performance metrics of PR in patients with COPD and the future optimisation of the impact of PR. Healthcare professionals and payers need to start realising that patients with COPD will respond differentially on the PR outcome measures that are regularly used [1]. Choosing only one or two outcomes as key performance indicators (e.g., exercise performance and health status) seems to ignore the clinical complexity of rehabilitating patients with COPD. The time has come to start using multidimensional outcome profiling to identify the right COPD patients for the right PR programme.

\section{Acknowledgements}

The CIRO+ Rehabilitation Network consists of: CIRO+ (Center of Expertise for Chronic Organ Failure) in Horn, St Laurentius Hospital in Roermond, St Jans Gasthuis in Weert, Catharina Hospital in Eindhoven, Maastricht University Medical Center (MUMC+) in Maastricht, St Anna Hospital in Geldrop and Elkerliek Hospital in Helmond (all in the Netherlands).

\section{References}

1 Spruit MA, Singh SJ, Garvey C, et al. An official American Thoracic Society/European Respiratory Society statement: key concepts and advances in pulmonary rehabilitation. Am J Respir Crit Care Med 2013; 188: el3-e64.

2 Lacasse Y, Goldstein R, Lasserson TJ, et al. Pulmonary rehabilitation for chronic obstructive pulmonary disease. Cochrane Database Syst Rev 2006; 4: CD003793.

3 Nici L, ZuWallack R. An official American Thoracic Society workshop report: the Integrated Care of The COPD Patient. Proc Am Thorac Soc 2012; 9: 9-18.

4 Spruit MA, Pitta F, Garvey C, et al. Differences in content and organisational aspects of pulmonary rehabilitation programmes. Eur Respir J 2014; 43: 1326-1337.

5 Troosters T, Gosselink R, Decramer M. Exercise training in COPD: how to distinguish responders from nonresponders. J Cardiopulm Rehabil 2001; 21: 10-17. 
Walsh JR, McKeough ZJ, Morris NR, et al. Metabolic disease and participant age are independent predictors of response to pulmonary rehabilitation. J Cardiopulm Rehabil Prev 2013; 33: 249-256.

7 Scott AS, Baltzan MA, Fox J, et al. Success in pulmonary rehabilitation in patients with chronic obstructive pulmonary disease. Can Respir J 2010; 17: 219-223.

8 de Torres JP, Pinto-Plata V, Ingenito E, et al. Power of outcome measurements to detect clinically significant changes in pulmonary rehabilitation of patients with COPD. Chest 2002; 121: 1092-1098.

9 Garrod R, Marshall J, Barley E, et al. Predictors of success and failure in pulmonary rehabilitation. Eur Respir J 2006; 27: 788-794.

10 Garrod R, Ford K, Daly C, et al. Pulmonary rehabilitation: analysis of a clinical service. Physiother Res Int 2004; 9: 111-120.

11 Crisafulli E, Costi S, Luppi F, et al. Role of comorbidities in a cohort of patients with COPD undergoing pulmonary rehabilitation. Thorax 2008; 63: 487-492.

12 Harrison SL, Greening NJ, Williams JE, et al. Have we underestimated the efficacy of pulmonary rehabilitation in improving mood? Respir Med 2012; 106: 838-844.

13 Laviolette L, Bourbeau J, Bernard S, et al. Assessing the impact of pulmonary rehabilitation on functional status in COPD. Thorax 2008; 63: 115-121.

14 Spruit MA, Gosselink R, Troosters T, et al. Low-grade systemic inflammation and the response to exercise training in patients with advanced COPD. Chest 2005; 128: 3183-3190.

15 Sewell L, Singh SJ, Williams JE, et al. Can individualized rehabilitation improve functional independence in elderly patients with COPD? Chest 2005; 128: 1194-1200.

16 Spruit MA, Vanderhoven-Augustin I, Janssen PP, et al. Integration of pulmonary rehabilitation in COPD. Lancet 2008; 371: 12-13.

17 van't Hul A, Gosselink R, Kwakkel G. Constant-load cycle endurance performance: test-retest reliability and validity in patients with COPD. J Cardiopulm Rehabil 2003; 23: 143-150.

18 Hernandes NA, Wouters EF, Meijer K, et al. Reproducibility of 6-minute walking test in patients with COPD. Eur Respir J 2011; 38: 261-267.

19 Holland AE, Spruit MA, Troosters T, et al. An official European Respiratory Society/American Thoracic Society technical standard: field walking tests in chronic respiratory disease. Eur Respir J 2014; 44: 1428-1446.

20 Singh SJ, Puhan MA, Andrianopoulos V, et al. An official systematic review of the European Respiratory Society/ American Thoracic Society: measurement properties of field walking tests in chronic respiratory disease. Eur Respir J 2014; 44: 1447-1478.

21 Annegarn J, Meijer K, Passos VL, et al. Problematic activities of daily life are weakly associated with clinical characteristics in COPD. J Am Med Dir Assoc 2012; 13: 284-290.

22 Sewell L, Singh SJ. The Canadian Occupational Performance Measure: is it a reliable measure in clients with chronic obstructive pulmonary disease? Br J Occup Ther 2001; 64: 305-310.

23 Zigmond AS, Snaith RP. The hospital anxiety and depression scale. Acta Psychiatr Scand 1983; 67: 361-370.

24 Jones PW, Quirk FH, Baveystock CM, et al. A self-complete measure of health status for chronic airflow limitation. The St. George's Respiratory Questionnaire. Am Rev Respir Dis 1992; 145: 1321-1327.

25 Vanfleteren LE, Spruit MA, Groenen MT, et al. Arterial stiffness in patients with COPD: the role of systemic inflammation and the effects of pulmonary rehabilitation. Eur Respir J 2014; 43: 1306-1315.

26 Sillen MJ, Franssen FM, Delbressine JM, et al. Efficacy of lower-limb muscle training modalities in severely dyspnoeic individuals with COPD and quadriceps muscle weakness: results from the DICES trial. Thorax 2014; 69: 525-531.

27 Vanfleteren LE, Spruit MA, Groenen M, et al. Clusters of comorbidities based on validated objective measurements and systemic inflammation in patients with chronic obstructive pulmonary disease. Am J Respir Crit Care Med 2013; 187: 728-735.

28 Puhan MA, Frey M, Buchi S, et al. The minimal important difference of the hospital anxiety and depression scale in patients with chronic obstructive pulmonary disease. Health Qual Life Outcomes 2008; 6: 46.

29 Jones PW. Interpreting thresholds for a clinically significant change in health status in asthma and COPD. Eur Respir J 2002; 19: 398-404.

30 Ries AL, Make BJ, Lee SM, et al. The effects of pulmonary rehabilitation in the national emphysema treatment trial. Chest 2005; 128: 3799-3809.

31 Spruit MA, Gosselink R, Troosters T, et al. Resistance versus endurance training in patients with COPD and peripheral muscle weakness. Eur Respir J 2002; 19: 1072-1078.

32 Sillen MJ, Franssen FM, Delbressine JM, et al. Heterogeneity in clinical characteristics and co-morbidities in dyspneic individuals with COPD GOLD D: findings of the DICES trial. Respir Med 2013; 107: 1186-1194.

33 Spruit MA, Pennings HJ, Janssen PP, et al. Extra-pulmonary features in COPD patients entering rehabilitation after stratification for MRC dyspnea grade. Respir Med 2007; 101: 2454-2463.

34 Agusti A, Calverley PM, Celli B, et al. Characterisation of COPD heterogeneity in the ECLIPSE cohort. Respir Res 2010; 11: 122.

35 Bowden MG, Behrman AL, Neptune RR, et al. Locomotor rehabilitation of individuals with chronic stroke: difference between responders and nonresponders. Arch Phys Med Rehabil 2013; 94: 856-862.

36 Schmid JP, Zurek M, Saner H. Chronotropic incompetence predicts impaired response to exercise training in heart failure patients with sinus rhythm. Eur J Prevent Cardiol 2013; 20: 585-592.

37 Cecchi F, Pasquini G, Paperini A, et al. Predictors of response to exercise therapy for chronic low back pain: result of a prospective study with one year follow-up. Eur J Phys Rehabil Med 2014; 50: 143-151.

38 Jones PW, Donohue JF, Nedelman J, et al. Correlating changes in lung function with patient outcomes in chronic obstructive pulmonary disease: a pooled analysis. Respir Res 2011; 12: 161.

39 Burge PS, Calverley PM, Jones PW, et al. Prednisolone response in patients with chronic obstructive pulmonary disease: results from the ISOLDE study. Thorax 2003; 58: 654-658.

40 Nonoyama ML, Brooks D, Guyatt GH, et al. Effect of oxygen on health quality of life in patients with chronic obstructive pulmonary disease with transient exertional hypoxemia. Am J Respir Crit Care Med 2007; 176: $343-349$. 
41 Deslee G, Klooster K, Hetzel M, et al. Lung volume reduction coil treatment for patients with severe emphysema: a European multicentre trial. Thorax 2014; 69: 980-986.

42 Fishman A, Martinez F, Naunheim K, et al. A randomized trial comparing lung-volume-reduction surgery with medical therapy for severe emphysema. N Engl J Med 2003; 348: 2059-2073.

43 Flores M, Glusman G, Brogaard K, et al. P4 medicine: how systems medicine will transform the healthcare sector and society. Per Med 2013; 10: 565-576.

44 Mesquita R, Vanfleteren LE, Franssen FME, et al. Objectively identified comorbidities in COPD: impact on pulmonary rehabilitation outcomes. Eur Respir J 2015; 46: 545-548. 